\title{
A Note on the Poincaré Inequality for Convex Domains
}

\author{
M. Bebendorf
}

\begin{abstract}
In this article a proof for the Poincaré inequality with explicit constant for convex domains is given. This proof is a modification of the original proof [5], which is valid only for the two-dimensional case.
\end{abstract}

Keywords: Poincaré inequality, convex domains

AMS subject classification: 26D10

\section{Introduction}

The classical proof for the Poincaré inequality

$$
\|u\|_{L^{2}(\Omega)} \leq c_{\Omega}\|\nabla u\|_{L^{2}(\Omega)}
$$

where $\Omega \subset \mathbb{R}^{n}$ is a bounded domain and $u \in H^{1}(\Omega)$ with vanishing mean value over $\Omega$, is based on the compact embedding of $H^{1}(\Omega)$ in $L^{2}(\Omega)$ which is valid under quite general assumptions on $\Omega$ (cf. [6]). However, the constant $c_{\Omega}$ depends on the domain $\Omega$, and the proof based on compactness does not provide insight into this dependency.

For practical purposes it is important to know an explicit expression for this constant (see, for example, $[2,7]$ ). Therefore, the special case of convex domains is interesting, since in [5] this constant is proved to be $\frac{d}{\pi}$, where $d$ is the diameter of $\Omega$. Though this proof is elegant, it contains a mistake in the case $n \geq 3$. The same mistake can also be found in [1], in which the $L^{1}$-estimate is considered.

The goal of this article is to fix this gap (see Remark 3.3). Luckily, the constant $\frac{d}{\pi}$ in the Poincaré inequality remains valid.

M. Bebendorf: Math. Inst. der Univ., Augustuspl. 10/11, D-04109 Leipzig; bebendorf@mathematik.uni-leipzig.de

ISSN 0232-2064 / \$2.50 C Heldermann Verlag Berlin 


\section{The one-dimensional case}

We first prove the Poincaré inequality for the one-dimensional case. In fact we will prove a generalization which the multi-dimensional case can be reduced to.

Lemma 2.1. Let $m \in \mathbb{N}$ and $\rho$ be a non-negative concave function on the interval $[0, L]$. Then, for all $u \in H^{1}(0, L)$ satisfying

$$
\int_{0}^{L} \rho^{m}(x) u(x) d x=0,
$$

there holds

$$
\int_{0}^{L} \rho^{m}(x)|u(x)|^{2} d x \leq \frac{L^{2}}{\pi^{2}} \int_{0}^{L} \rho^{m}(x)\left|u^{\prime}(x)\right|^{2} d x .
$$

Furthermore, the constant $\frac{L^{2}}{\pi^{2}}$ is optimal.

\section{Proof.}

(a) Let us first assume that $\rho$ is strictly positive and twice differentiable. Then each non-zero function $v$ minimizing the quotient

$$
\frac{\int_{0}^{L} \rho^{m}(x)\left|u^{\prime}(x)\right|^{2} d x}{\int_{0}^{L} \rho^{m}(x)|u(x)|^{2} d x}
$$

and satisfying (2.1) must satisfy the Sturm-Liouville system (cf. [3])

$$
\left.\begin{array}{r}
{\left[\rho^{m} v^{\prime}\right]^{\prime}+\lambda \rho^{m} v=0} \\
v^{\prime}(0)=v^{\prime}(L)=0
\end{array}\right\}
$$

where $\lambda$ is the minimum of quotient (2.3). After dividing (2.4) by $\rho^{m}$ and differentiating, we introduce the new variable $w=\rho^{m / 2} v^{\prime}$ and obtain

$$
\left.\begin{array}{r}
w^{\prime \prime}+\frac{m}{2}\left[\frac{\rho^{\prime \prime}}{\rho}-\left(1+\frac{m}{2}\right) \frac{\left(\rho^{\prime}\right)^{2}}{\rho^{2}}\right] w+\lambda w=0 \\
w(0)=w(L)=0
\end{array}\right\} .
$$

Since $\rho$ is concave, $\rho^{\prime \prime} \leq 0$. Hence, $w^{\prime \prime}+(\lambda-a) w=0$, where $a$ is a non-negative function. Integration by parts leads to

$$
\lambda \geq \frac{\int_{0}^{L}\left|w^{\prime}(x)\right|^{2} d x}{\int_{0}^{L}|w(x)|^{2} d x} .
$$


The last quotient is bounded by the first eigenvalue of the vibrating string with fixed ends, which gives $\lambda \geq \frac{\pi^{2}}{L^{2}}$.

(b) If $\rho$ is a non-negative concave function, we may represent it as the $L^{\infty}$-limit of strictly positive concave $C^{2}$-functions $\rho_{k}$ (cf. [4]). From Part (a) one has

$$
\int_{0}^{L} \rho_{k}^{m}(x)|\hat{u}(x)|^{2} d x \leq \frac{L^{2}}{\pi^{2}} \int_{0}^{L} \rho_{k}^{m}(x)\left|u^{\prime}(x)\right|^{2} d x
$$

where $\hat{u}(x)=u(x)-\bar{u}$ and

$$
\bar{u}=\frac{\int_{0}^{L} \rho_{k}^{m}(x) u(x) d x}{\int_{0}^{L} \rho_{k}^{m}(x) d x} .
$$

Hence,

$$
\int_{0}^{L} \rho_{k}^{m}(x)|u(x)|^{2} d x \leq \frac{L^{2}}{\pi^{2}} \int_{0}^{L} \rho_{k}^{m}(x)\left|u^{\prime}(x)\right|^{2} d x+\bar{u} \int_{0}^{L} \rho_{k}^{m}(x) u(x) d x .
$$

In the limit $k \rightarrow \infty$ we obtain (2.2).

(c) To see that the constant $\frac{L^{2}}{\pi^{2}}$ is optimal, choose $\rho^{m} \equiv 1, L=1$ and $u(x)=\cos (\pi x)$. Then $\int_{0}^{1} \rho^{m}(x) u(x) d x=0$ and

$$
\frac{\int_{0}^{1} \rho^{m}(x)|u(x)|^{2} d x}{\int_{0}^{1} \rho^{m}(x)\left|u^{\prime}(x)\right|^{2} d x}=\frac{1}{\pi^{2}} \frac{\int_{0}^{1} \cos ^{2}(\pi x) d x}{\int_{0}^{1} \sin ^{2}(\pi x) d x}=\frac{1}{\pi^{2}} .
$$

Thus the lemma is proved

\section{The $n$-dimensional case}

In the rest of this article we will consider the case $n \geq 2$. By the following lemma we are able to reduce the $n$-dimensional problem to the one-dimensional case.

Lemma 3.1. Let $\Omega \subset \mathbb{R}^{n}$ be a convex domain with diameter $d$. Assume that $u \in L^{1}(\Omega)$ satisfies $\int_{\Omega} u(x) d x=0$. Then for any $\delta>0$ there are disjoint convex domains $\Omega_{i} \quad(i=1, \ldots, k)$ such that

$$
\bar{\Omega}=\bigcup_{i=1}^{k} \bar{\Omega}_{i}, \quad \int_{\Omega_{i}} u(x) d x=0
$$


and for each $\Omega_{i}$ there is rectangular coordinate system such that

$$
\Omega_{i} \subset\left\{(x, y) \in \mathbb{R}^{n}: 0 \leq x \leq d \text { and }\left|y_{j}\right| \leq \delta, j=1, \ldots, n-1\right\} .
$$

Proof. For each $\alpha \in[0,2 \pi]$ there is a unique hyperplane $H_{\alpha} \subset \mathbb{R}^{n}$ with normal $(0, \ldots, 0, \cos (\alpha), \sin (\alpha))$ that divides $\Omega$ into two convex sets $\Omega_{\alpha}^{\prime}$ and $\Omega_{\alpha}^{\prime \prime}$ of equal volume. Since $I(\alpha)=-I(\alpha+\pi)$, where $I(\alpha)=\int_{\Omega_{\alpha}^{\prime}} u(x) d x$, by continuity there is $\alpha_{0}$ such that $I\left(\alpha_{0}\right)=0$. Applying this procedure recursively to each of the parts $\Omega_{\alpha_{0}}^{\prime}$ and $\Omega_{\alpha_{0}}^{\prime \prime}$, we are able to subdivide $\Omega$ into convex sets $\Omega_{i}$ such that each of the sets is contained between two parallel hyperplanes with normal of the form $(0, \ldots, 0, \cos (\beta), \sin (\beta))$ at distance at most $\delta$, and the average of $u$ vanishes on each of them.

Consider one of these sets. By rotating the coordinate system we can assume that the normal of the enclosing hyperplanes is $(0, \ldots, 0,1)$. In these coordinates we apply the above arguments using hyperplanes with normals of the form $(0, \ldots, 0, \cos (\alpha), \sin (\alpha), 0)$. Continuing this procedure we end up with the desired decomposition of $\Omega$

Theorem 3.2. Let $\Omega \subset \mathbb{R}^{n}$ be a convex domain with diameter $d$. Then

$$
\|u\|_{L^{2}(\Omega)} \leq \frac{d}{\pi}\|\nabla u\|_{L^{2}(\Omega)}
$$

for all $u \in H^{1}(\Omega)$ satisfying $\int_{\Omega} u(x) d x=0$.

Proof. Let us first assume that $u$ is twice continuously differentiable. According to the previous Lemma 3.1 we are able to decompose $\Omega$ into convex subsets $\Omega_{i}$ such that for each $\Omega_{i}$ there is a rectangular coordinate system in which $\Omega_{i}$ is contained in

$$
\left\{(x, y) \in \mathbb{R}^{n}: 0 \leq x \leq d_{i} \text { and }\left|y_{j}\right| \leq \delta, j=1, \ldots, n-1\right\}
$$

We may assume that the interval $\left[0, d_{i}\right]$ on the $x$-axis is contained in $\Omega_{i}$. Let $R(t)$ be the $(n-1)$-volume of the intersection of $\Omega_{i}$ with the hyperplane $x=t$. In polar coordinates $R(t)$ can be written in the form

$$
R(t)=\int_{\mathbb{S}^{n-2}} \int_{0}^{\rho(t, \omega)} r^{n-2} d r d \omega=\frac{1}{n-1} \int_{\mathbb{S}^{n-2}} \rho^{n-1}(t, \omega) d \omega
$$

where $\rho(t, \omega)$ is the distance of the boundary point of $\Omega_{i}$ at $(t, \omega)$ to the $x$-axis. Since $\Omega_{i}$ is convex, $\rho$ is a concave function with respect to $t$. 
From the smoothness of $u$ it can be seen that there are constants $c_{1}, c_{2}$ and $c_{3}$ such that

$$
\begin{aligned}
\left|\int_{\Omega_{i}} u(x, y) d x d y-\int_{0}^{d_{i}} u(x, 0) R(x) d x\right| & \leq c_{1}\left|\Omega_{i}\right| \delta \\
\left.\left|\int_{\Omega_{i}}\right| \frac{\partial u}{\partial x}(x, y)\right|^{2}-\int_{0}^{d_{i}}\left|\frac{\partial u}{\partial x}(x, 0)\right|^{2} R(x) d x \mid & \leq c_{2}\left|\Omega_{i}\right| \delta \\
\left.\left|\int_{\Omega_{i}}\right| u(x, y)\right|^{2} d x d y-\int_{0}^{d_{i}}|u(x, 0)|^{2} R(x) d x \mid & \leq c_{3}\left|\Omega_{i}\right| \delta .
\end{aligned}
$$

Let $\omega \in \mathbb{S}^{n-2}$. Since $u(\cdot, 0) \in H^{1}\left(0, d_{i}\right)$, we can apply Lemma 2.1 to $\hat{u}_{\omega}(x):=$ $u(x, 0)-\bar{u}_{\omega}$, where

$$
\bar{u}_{\omega}:=\frac{\int_{0}^{d_{i}} u(x, 0) \rho^{n-1}(x, \omega) d x}{\int_{0}^{d_{i}} \rho^{n-1}(x, \omega) d x} .
$$

Hence,

$$
\int_{0}^{d_{i}}\left|\hat{u}_{\omega}(x)\right|^{2} \rho^{n-1}(x, \omega) d x \leq \frac{d_{i}^{2}}{\pi^{2}} \int_{0}^{d_{i}}\left|\frac{\partial u}{\partial x}(x, 0)\right|^{2} \rho^{n-1}(x, \omega) d x .
$$

Applying Fubini's theorem we obtain

$$
\begin{aligned}
\frac{d_{i}^{2}}{\pi^{2}} \int_{0}^{d_{i}} & \left|\frac{\partial u}{\partial x}(x, 0)\right|^{2} R(x) d x \\
& =\frac{1}{n-1} \int_{\mathbb{S}^{n-2}} \frac{d_{i}^{2}}{\pi^{2}} \int_{0}^{d_{i}}\left|\frac{\partial u}{\partial x}(x, 0)\right|^{2} \rho^{n-1}(x, \omega) d x d \omega \\
& \geq \frac{1}{n-1} \int_{\mathbb{S}^{n-2}} \int_{0}^{d_{i}}\left|\hat{u}_{\omega}(x)\right|^{2} \rho^{n-1}(x, \omega) d x d \omega \\
& =\frac{1}{n-1} \int_{\mathbb{S}^{n-2}} \int_{0}^{d_{i}} \hat{u}_{\omega}(x) u(x, 0) \rho^{n-1}(x, \omega) d x d \omega \\
& \geq \int_{0}^{d_{i}}|u(x, 0)|^{2} R(x) d x-M\left|\int_{0}^{d_{i}} u(x, 0) R(x) d x\right|
\end{aligned}
$$

where $M=\max _{\omega \in \mathbb{S}^{n-2}}\left|\bar{u}_{\omega}\right|$. By $\int_{\Omega_{i}} u(x, y) d x d y=0$, (3.2) and (3.3) we are lead to

$$
\begin{aligned}
\int_{0}^{d_{i}}|u(x, 0)|^{2} R(x) d x & \leq \frac{d_{i}^{2}}{\pi^{2}} \int_{0}^{d_{i}}\left|\frac{\partial u}{\partial x}(x, 0)\right|^{2} R(x) d x+c_{1}\left|\Omega_{i}\right| M \delta \\
& \leq \frac{d_{i}^{2}}{\pi^{2}} \int_{\Omega_{i}}|\nabla u(x, y)|^{2} d x d y+\left(c_{1} M+c_{2} \frac{d_{i}^{2}}{\pi^{2}}\right)\left|\Omega_{i}\right| \delta .
\end{aligned}
$$


From (3.4) and the summation over $i$ we obtain

$$
\int_{\Omega}|u(x, y)|^{2} d x d y \leq \frac{d^{2}}{\pi^{2}} \int_{\Omega}|\nabla u(x, y)|^{2} d x d y+\left(c_{1} M+c_{2} \frac{d^{2}}{\pi^{2}}+c_{3}\right)|\Omega| \delta
$$

and, since $\delta>0$ is arbitrary, the desired estimate is proven. The assertion follows from the density of $C^{\infty}(\bar{\Omega})$ in $H^{1}(\Omega)$

Remark 3.3. In [5] it is claimed that $R$ from (3.1) is a concave function, which is not true for $n \geq 3$ as can be seen from the following example. Let

$$
\Omega=\left\{\left(x_{1}, x_{2}, x_{3}\right) \in \mathbb{R}^{3}: \sqrt{x_{2}^{2}+x_{3}^{2}} \leq \frac{1}{2}\left(x_{1}+1\right), 0 \leq x_{1} \leq 1\right\}
$$

Then $\Omega$ is convex, vol $R(0)=\frac{\pi}{4}$ and $\operatorname{vol} R(1)=\pi$. But

$$
\operatorname{vol} R\left(\frac{1}{2}\right)=\frac{9}{16} \pi<\frac{5}{8} \pi=\frac{1}{2} \operatorname{vol} R(0)+\frac{1}{2} \operatorname{vol} R(1) \text {. }
$$

Hence, $R$ is not concave.

Acknowledgement. Thanks go to C. Carstensen for pointing out the mistake in [5].

\section{References}

[1] Acosta, G. and R. G. Durán: An optimal Poincaré inequality in $L^{1}$ for convex domains. Preprint. University of Buenos Aires 2003.

[2] Bebendorf, M. and W. Hackbusch: Existence of $\mathcal{H}$-matrix approximants to the inverse FE-matrix of elliptic operators with $L^{\infty}$-coefficients. Numer. Math. 2003 (to appear).

[3] Courant, R. and D. Hilbert: Methoden der mathematischen Physik, Band I. Dritte Auflage. Berlin: Springer-Verlag 1968.

[4] Eggleston, H. G.: Convexity. New York: Cambridge Univ. Press 1958.

[5] Payne, L. E. and H. F. Weinberger: An optimal Poincaré inequality for convex domains. Arch. Rat. Mech. Anal. 5 (1960), 286 - 292.

[6] Wloka, J.: Partial Differential Equations (translated from the German by C. B. Thomas and M. J. Thomas). Cambridge: Cambridge Univ. Press 1987.

[7] Verfürth, R.: A note on polynomial approximation in Sobolev spaces. Math. Model. Numer. Anal. 33 (1999), 715 - 719.

Received 28.02.2003 